\title{
Research on Predict Induction Ground Wire Producing
}

\author{
Jiang Guangmin ${ }^{1}$, Yuan Ting ${ }^{1}$, Ding Desheng ${ }^{2}$, Ding Yixin ${ }^{2}$, Han Lin ${ }^{2}$, Wu \\ Yaowei $^{2}$, Mu Jinglong ${ }^{2}$, Guan Chunmei ${ }^{2}$, Huang Hongda ${ }^{1}$, Qu Weisong ${ }^{1}$, Chen \\ Zhiheng $^{1}$, Jin Xiancheng ${ }^{1}$ \\ ${ }^{1}$ Panjin Power Supply Company, Liaoning Electric Power Company Limited, State Grid, China, \\ ${ }^{2}$ Fushun Power Supply Company, Liaoning Electric Power Company Limited, State Grid, China,
}

fushunpowersupply@163.com

Keywords: Mount ground, Body insulation, Induced voltage predicted, Caveat

\begin{abstract}
The current transmission line is constantly improved and updated, in order to save space, a lot of back and multi-line double back. Power lines during the operation induced voltage is very large, you must mount the ground. Ground wire must be multi-strand copper wire, whose cross-sectional area of not less than $25 \mathrm{~mm} 2$. General grounding wire with multi-strand copper surface put a layer of soft plastics, pipe insulation fight against human touch ground. In the winter, plastics, pipe breakage fold to fold to expose the soft copper wire, to bring workers considerable danger. To solve this technical problem, the development of transmission lines "predict induction grounding line" means the use of the device can be realized with the tower and rack (or parallel) operations induced voltage power lines wounding problems. Also explained the harm previous grounding line, and discusses the "predict induction grounding line," the theoretical basis and the main components.
\end{abstract}

\section{Introduction}

With the development of the power system, is limited by the line corridors (land, space), with the tower and rack (or parallel) transmission lines more and more. Either zero-sequence current loop occurs; it will produce a certain amount of alternating magnetic flux around. Since the magnetic coupling causes and frame (or parallel) of the second loop induced voltage is generated, a large mutual inductance voltage up to $30 \mathrm{KV}$, mutual inductance current up to 50A. Often occurs because the induced voltage resulting in death and serious injury. In order to avoid such accidents, power transmission lines in operation, the suspension is to ensure the safety of the ground wire indispensable link[1-3].

Conventional ground wire, ground chuck is screw; wire end is a spring hook, intermediate connecting soft copper wire. Grounding wire with multi-strand soft copper surface layer of insulating sleeve stroke plastics, tubes, plastics, pipe bending in the winter easily damaged soft copper exposed. "Safety Regulations" provisions, in order to prevent the body hit the ground wire directly in the suspension process, workers are not allowed to directly hand grasping soft copper wire. Many workers do not operate according to procedures required, but directly with the clutch soft copper wire, to bring workers at risk. Original ground wire is soft structure, inconvenient in suspension; the hook is likely to fall. Especially in windy weather and more prone is to accidents when personal sense of power transmission line tower and rack to hang with the ground. For a comprehensive analysis and comparison of the above, decided to develop predictable - Sensing ground wire[4].

\section{Conventional ground operation hazards}

Conventional ground wire from ground clip, section not less than 25 square copper wire and ground wire threaded chuck components. Used to clamp attached to the wire. Ground screw clamp fixed on the angle iron. During equipment maintenance, by workers on the ground wire is attached to electrical equipment near the wire. When the lines of a one wire power is outage, the wire by the 
electric field near the wire will produce EMF. If the ground wire hangs properly, the existence of the phenomenon of virtual access, resulting in the grounding wire can not be reliably grounded. Induction is bound to cause harm to workers. According to reports in 2008, Yichang, Hubei Power Company EHV Transmission at the time of the $500 \mathrm{kV}$ line towers were cleaning insulators and eliminating defects jobs chest close to the ground, a worker ground terminal and a sense of power and death. Currently used ground wire (copper wire) hanging in the air, shaking amplitude, in particular the use of the tower erected on the same line, the distance is difficult to ensure safety[5].

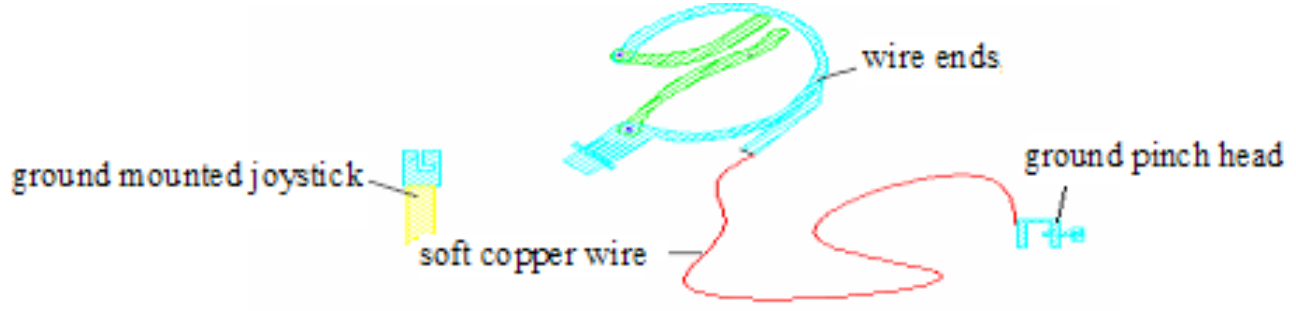

Figure 1. A schematic view of a conventional ground wire

\section{Predict Induction the advantages of the ground wire}

(1) Induced voltage alarm

Grounding wire can not solve the induced voltage alarm, there are safety problems. The following technical solutions are proposed. This predictable induction grounding line, which includes no less than $25 \mathrm{~mm} 2$ cross-section of soft copper wire and connect one end of the ground clamp, ground screw clamp the other end of the connection, and its special features are installed on the ground clip induced voltage alarm device.

(2) Prevent shaking

In order to prevent cross-section of not less than $25 \mathrm{~mm} 2$ copper wire in the air shaking big problem. The cross section of not less than $25 \mathrm{~mm} 2$ copper wire through the insulating telescopic operating rod, at its tip is connected to the clamp, threaded at its terminus ground chuck.

(3) Convenient retractable soft copper wire

In order to facilitate the retractable section is not less than $25 \mathrm{~mm} 2 \mathrm{soft}$ copper, even in insulated panels installed at the drum telescopic operating rod ends. The end of the cross-section of not less than $25 \mathrm{~mm} 2$ soft copper Chan around the drum. A period of not less than $25 \mathrm{~mm} 2$ cross section of soft copper wire, one end of the connection link plate, the other end is connected ground thread chuck.

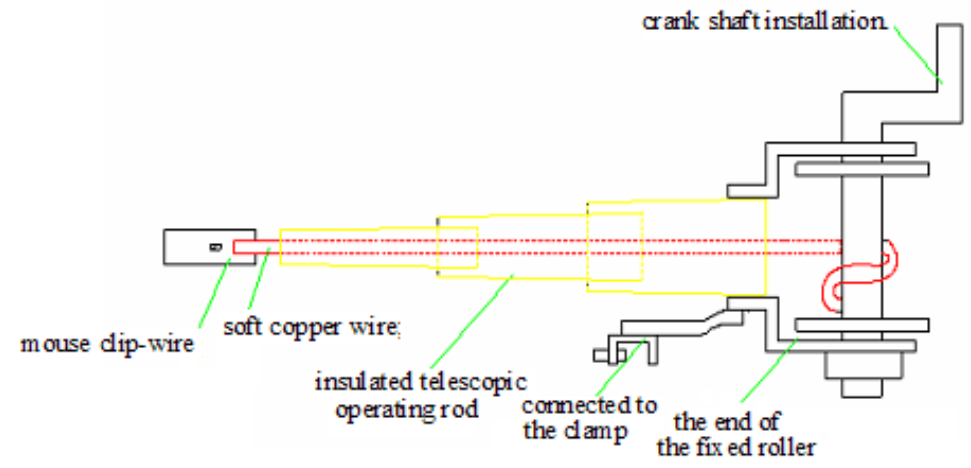

Figure 2 predict the structure of the ground wire sensors

\section{Predict Induction Ground wire composition}

(1) Predict Induction Ground wire composition

Predict - Induction - mainly by rat grounding clip-wire portion of the chuck, $25 \mathrm{~mm} 2$ copper wire, insulated telescopic operating lever to clamp after connecting other components, as shown in Figure 2.

(2) Alarm device 
Predict induction ground wire is the most important part mousetrap type wire ends chuck installed alarm devices. Alarm apparatus are shown in Figure 3. Alarm device include BG1, BG2 and other external components, oscillators, BG3 and other external components, trigger circuit configuration. When a charged object near the grounding line, BG3 conduction, BG2 base has a high potential oscillation circuit, the speaker sounds the alarm. Figure $3 \mathrm{~K} 1$ to select different resistance, may constitute $10 \mathrm{kV}, 66 \mathrm{kV}, 220 \mathrm{kV}$ different voltage levels. AN is the test button, circuit sound when turned on, you can verify that the circuit is working properly. AN When turned on, the capacitor C3 is charged, oscillator, Y horn sound. When AN disconnect capacitors to discharge into a certain voltage, the oscillator is stopped.

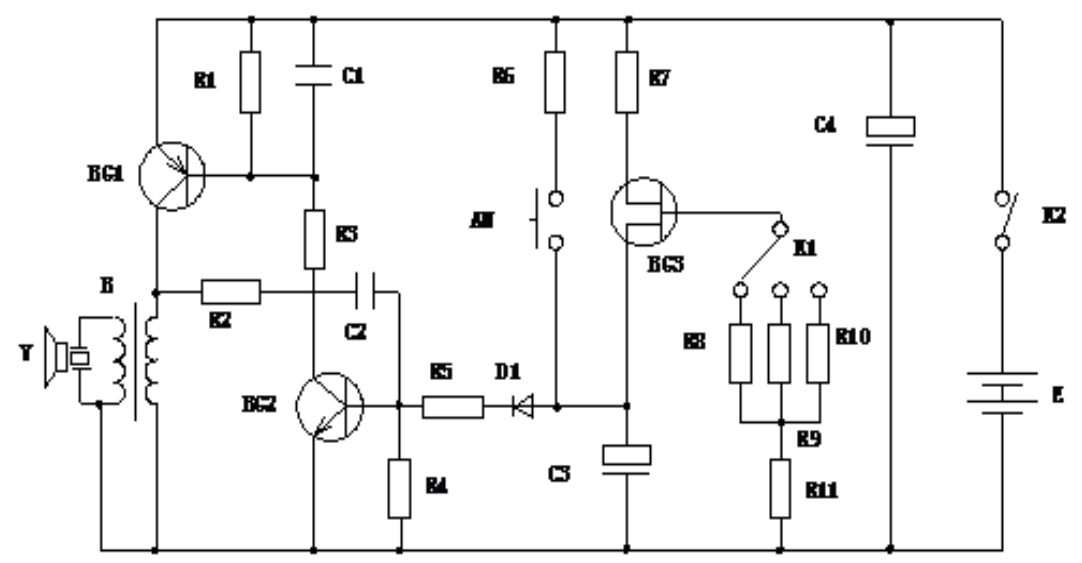

Figure 3 Wire ends mousetrap alarm devices installed on the wiring diagram

\section{Predict Induction grounding function}

Predict induction grounding wire, can solve the problem of the induced voltage alarms. Soft copper wire at one end of the ground wire to predict induction ground clip, and the other end connected to the ground screw chuck. After installing the ground clip induced voltage alarm, soft copper wire through the insulation telescopic operating rod, at its tip is connected to the clamp, threaded connection ground chuck at its end. At the end of the insulated operating rod mounted telescopic cylinder via the link plate. Soft copper wire, connected to the first end plate is connected to the end of the thread connecting the ground chuck. Avoid predictable induction ground wire grounding wire is bad when used to solve wire (ground) of the induced voltage on the workers a sense of power accident. Since the operating lever multi-section telescopic pipe insulation sleeve-type structure, can prevent the body soft touch multi-strand copper wire. At the end of the operating lever mounted roller, thus facilitating the collection and carry.

\section{Predict Induction grounding effect}

For example, April 2, 201408 to 00 minutes at 16 o'clock on the April 2, 2014, the main line operations: $220 \mathrm{kV}$ line North 51 days, No. 95, ministries inspection, vibration reset. Retention of live parts: $220 \mathrm{kV}$ North-day-wire (right red line) has power, located to the right direction for the increased number of tower. No. 96-day power outage in North $220 \mathrm{kV}$ line No. 95 with the tower was erected. Responsible person check on the construction job site, no problem, make sure that all personnel under the tower after all, ordered No. 51 and No. 95 to install two sets of ground wire. First of all, connect the ground wire to predict ground sensors, and then pulled out a section telescopic pipe insulation, pipe insulation operator holding the rear, when close to the ground wire conductor predict sensing alarm device alarm sound, proven high induced voltage wires, thanks to predict induction grounding line, managed to avoid the sense of power injury accident. 


\section{In conclusion}

Predict induction grounding wire, easy operation, small size, light weight (5 kg). It can be a single person operation, predict the induction of the ground wire to ground effect, a large contact area. It can predict the induced voltage, while the poor are able to contact the police. By improving reliability grounding, the operator does not touch the bare copper wire, to protect the personal safety of operating personnel.

\section{References}

[1]Zhang Longcai, Jiang Xipei, You Chuanyong. Status and prospects of new composite materials composite core wire. China Electric Power Press, 2005.

[2]You Chuanyong. A Comparative Study of overhead transmission line conductors Capacity [J]. Power construction, 2007.

[3]Zhao Zuoli, Wang Li, Jiang Zhenfu. Carbon fiber composite core aluminum wire used in the transformation of the grid lines in Liaoning [J]. Power construction, 2007.

[4]Zhang Rui, Wu Yaguang. Development and Prospect of China's key manufacturing technology of composite insulators [J]. High Voltage Technology, 2007.

[5]Ding Jingling. Research Rod Composite Insulator Metal accessory connection zone mechanical properties [J]. Porcelain arrester, 2006. 